\title{
Testando a Assimetria nos Preços da Gasolina Brasileira
}

\author{
Carlos Frederico Azeredo Uchôa*
}

Sumário: 1. Introdução; 2. Algumas Justificativas Teóricas para a Existência de Assimetria; 3. Método de Investigação; 4. Análise Empírica; 5. Considerações Finais.

Palavras-chave: Co-Integração; Modelos Não-Lineares; Preço da Gasolina.

Códigos JEL: C50, C15, D12.

Este trabalho tem como objetivo desenvolver uma análise dos preços da gasolina brasileira, evidenciando uma relação de equilíbrio não-linear com os preços do petróleo no mercado internacional e com a taxa de câmbio. Os resultados mostram que preços da gasolina recuperam, em média, $90 \%$ das discrepâncias negativas de um período para outro. No entanto, apenas $5 \%$ das diferenças positivas são ajustadas, ou ainda não são recuperadas.

This paper has the objective to present an analysis of the prices in the Brazilian retail gasoline market, evidencing a non-linear relationship with the international oil prices and the Brazilian exchange rate. The results show that the gasoline price recover, on average, 90\% of the negative discrepancies from a period to other. However, only 5\% positive differences are adjusted, or still are not recovered.

\section{INTRODUÇÃO}

O comportamento dos preços do petróleo no comércio internacional influencia, de forma decisiva, os preços de seus derivados na maioria das nações. Mesmo num país como o Brasil onde as importações em 2003 responderam por apenas $9 \%$ do que é consumido internamente, os preços da gasolina não ficam indiferentes às variações dos preços do petróleo no mercado mundial Petrobrás (2005).

Uma trajetória de alta no preço do petróleo é acompanhada de perto por toda a sociedade, esperando seus reflexos imediatos no nível de preços dos combustíveis, sendo que, um dos impactos mais sentidos é, sem dúvida, sobre a gasolina. Mas, o que ocorre quando o sentido é inverso, isto é, e quando há uma queda nos preços do petróleo?

A questão que se coloca neste caso é sobre o tipo de ajustamento observado nos preços da gasolina. E não é difícil perceber que estes não ocorrem de maneira simétrica. Uma piora no cenário econômico, ou seja, a elevação na cotação dos preços petróleo e/ou desvalorização do câmbio, por exemplo, não demora muito para começar a pressionar por um aumento nos preços dos combustíveis no mercado

* Mestrando em Economia do CME/UFBA. Praça 13 de Maio n. 6, $5^{\circ}$ andar, CEP 40070-010 - Salvador, BA. E-mail: uchoa@email. com. 
interno. Porém, quando há uma melhora, o retorno ao nível anterior é lento e, na maioria das vezes, sequer ocorre.

O tanque do automóvel é abastecido constantemente, e os preços da gasolina são afixados em cada posto. Conseqüentemente, a maioria dos consumidores está ciente dos movimentos nos preços da gasolina e observam essa. É comum, consumidores queixarem-se que a ascensão dos preços da gasolina se dá mais rapidamente quando os preços do petróleo estão subindo do que quando ocorre o contrário. Este comportamento exibe uma relação assimétrica que pode ser vista com facilidade pelas pessoas.

Sendo assim, para ratificar as suspeitas dos consumidores é possível desenvolver uma análise dos movimentos nos preços da gasolina que permita comprovar esta suspeita. Neste sentido, pesquisadores têm investigado este fenômeno e as conclusões variam de acordo com o país em questão e com o método de análise utilizado. Mesmo nos casos em que se analisa um mesmo país os resultados podem diferir em função da escolha dos dados e/ou do método de estimação.

Até onde se possa perceber, não existe trabalho publicado no Brasil que acerca desta questão. Já na literatura internacional existem algumas referências importantes. O estudo de Borenstein et alii (1997) é, com certeza, um dos mais detalhados. Com uma série de modelos de correção de erros bivariados, os autores testam a existência de assimetria no movimento dos preços entre vários estágios da produção e da distribuição da gasolina nos Estados Unidos.

Neste estudo concluem que há forte evidência de assimetria em todos os segmentos do mercado. Suas conclusões foram no sentido de que os preços no mercado de varejo respondiam a um aumento nos preços do petróleo em quatro semanas, mas demoravam mais de oito semanas para responder a uma queda de igual magnitude. Da mesma maneira, Müller e Ray (2004) também encontraram evidências da presença de assimetria sistemática no ajustamento dos preços dos combustíveis no mercado americano.

Bachmeier e Griffin (2003) analisaram o mesmo problema e concluíram, de forma oposta, que não era possível assegurar a existência de assimetria. No entanto, suas conclusões basearam-se num modelo de correção de erros padrão em dois estágios como proposto originalmente por Engle e Granger (1987). Neste mesmo trabalho também foi utilizado o método proposto por Borenstein et alii (1997), sendo que, neste caso, usando dados diários, encontrou-se evidência de assimetria porém, insignificante.

Balke et alii (1998) obtiveram resultados ambíguos para suas estimativas que variavam de acordo com o método usado. Para o modelo com correção de erros, mas sem restrição ao equilíbrio de longo prazo, a assimetria mostrou-se alta. Num outro modelo foram utilizados parâmetros para o comportamento de alta e de baixa dos preços. Nos casos em que a assimetria mostrou-se estatisticamente significativa seu impacto na taxa de mudança dos preços da gasolina foi irrelevante. De forma geral o modelo com correção de erros ajustou-se melhor aos dados o que, segundo os autores sugeriria que a assimetria está relacionada com a taxa de mudança dos preços. Contudo, em se aceitando esta hipótese seria necessário admitir que os resultados têm relação com o modelo escolhido.

Radchenko (2004) através de um modelo ajustamento parcial estudou a relação entre a assimetria nos preços da gasolina e a volatilidade nos preços do petróleo mostrando que existe uma forte relação negativa entre os dois. Suas conclusões foram no sentido de que o grau de assimetria pode ser atribuído a uma rápida resposta dos preços da gasolina a um aumento da volatilidade nos preços do petróleo.

No mesmo sentido, para os mercados onde a taxa de cambio do dólar exerce algum tipo de influência na composição dos preços da gasolina as conclusões diferem significativamente entre si. Godby et alii (2000) concluíram pela não existência de assimetria no mercado canadense. Suas conclusões basearam-se num modelo de correção de erros assimétrico, na forma de um Threshold Auto-Regressive Model (TAR), para testar o ajustamento dos preços da gasolina.

Na Holanda, Bettendorf et alii (2003), não obtiveram um resultado preciso. Usando um modelo de correção de erros assimétrico, deduziram que a existência ou não de assimetria está ligada à data escolhida para a realização da observações. Assim, dependendo da estimativa das mudanças semanais e do dia escolhido, encontrou-se ou não evidências de um ajustamento assimétrico. Este resultado dúbio não encontrou uma explicação satisfatória na teoria econômica. 
Galeotti et alii (2003), estudando o mercado internacional, concluíram que Itália, Alemanha e Inglaterra não apresentavam qualquer tipo de assimetria na variação dos preços da gasolina, enquanto que França e Espanha a tinham, tanto na comercialização quanto na distribuição do combustivel.

Investigando a assimetria no mercado de gasolina das Filipinas, Salas (2002) estimou três modelos econométricos para o ajustamento dos preços da gasolina. As conclusões foram no sentido de que os preços nos postos de revenda respondem, de forma significativa, mais rapidamente a aumentos nos preços do petróleo do que a reduções.

O objetivo deste artigo vai na mesma direção dos trabalhos aqui mencionados e procura testar a hipótese de ajustamento assimétrico dos preços da gasolina no mercado brasileiro. Para tanto, na próxima seção serão apresentadas algumas explicações que tentam fornecer embasamento teórico para este fenômeno. Na terceira seção será apresentado o procedimento econométrico, através do qual pretende-se proceder a análise empírica. A seguir, na quarta seção, é feita a estimação das equações e discutido o desempenho e a adequação de cada uma delas. Por fim são feitas as Considerações Finais.

\section{ALGUMAS JUSTIFICATIVAS TEÓRICAS PARA A EXISTÊNCIA DE ASSIMETRIA}

Existem várias teorias que fornecem um embasamento teórico para o comportamento assimétrico dos preços da gasolina. O poder de mercado dos postos de revenda é provavelmente a explicação mais interessante para aqueles que observam a assimetria nos preços da gasolina. No entanto, ainda que a intuição possa atribuir a existência de assimetria ao poder de mercado, Peltzman (2000) mostrou que nos EUA estes dois fenômenos não têm ligação. Os custos que o consumidor tem na procura pelo menor preço e as vantagens na localização de alguns postos, podem fornecer certo poder do mercado aos varejistas, mas tal fato também pode ser visto como custos de diferenciação de produto sob a hipótese de competição monopolística Borenstein et alii (1997).

Seja, por exemplo, uma indústria com algumas firmas dominantes que agem em conluio para manter suas margens de lucro elevadas. Se as firmas tiverem conhecimento imperfeito sobre os preços que seus concorrentes estão pagando às distribuidoras então, cada firma tem uma função assimétrica de perda com a qual a firma é mais relutante em baixar seu preço de venda do que subi-lo. Sendo assim,

"This theory is based on the assumption that the observed asymmetry in the response of gasoline prices is evidence of imperfect competition among retailers. (...) In this case, price reduction occurs only if there is a significant drop in sales indicating price cutting by other retailers" Radchenko (2004).

Por outro lado, quando os preços das distribuidoras começam a subir, cada firma procura rapidamente levantar seu preço de venda sinalizando a seus concorrentes que está aderindo ao acordo tácito não diminuindo sua margem de lucro. Quando o preço das distribuidoras cai, cada firma é lenta em reduzir seus preços de venda porque isto pode ser interpretado como um sinal, por seus concorrentes, de que ela está diminuindo sua margem de lucro e assim tentando burlar o acordo.

Outra hipótese a ser levada em conta é a de que no mercado de varejo da gasolina, os custos de procura do consumidor poderiam conduzir ao poder provisório de mercado pelos postos de revenda e a uma resposta assimétrica às mudanças no preço por atacado como sugerem Borenstein et alii (1997) e Peltzman (2000). Neste caso, cada posto de gasolina teria um pequeno monopólio local que é limitado pela capacidade de procura do consumidor.

Isto ocorreria uma vez que, quando os preços por atacado sobem, os donos dos postos de combustivel procuram manter suas margens de lucro e repassam rapidamente o aumento para os preços de revenda. Quando os preços por atacado caem, cada posto mantém temporariamente suas margens de lucro repassando a diminuição lentamente aos clientes. O que só ocorreria depois que os clientes efetuassem uma nova pesquisa para encontrar preços menores. Somente neste estágio os postos de 
revenda seriam forçados a praticar preços a um nível competitivo com o que as margens de lucro em cada posto estariam num nível normal.

"The key notion in this line of thinking is that asymmetric adjustment presents competitors an opportunity to undercut the retailer's prices when costs go down.(...) Even if the consumers knew that a cheaper price was available elsewhere, it may not be worthwhile for them to switch retailers because there are costs involved in the transaction of switching, and learning about the new alternative" Müller e Ray (2004).

Portanto, o montante de dinheiro economizado é uma parte muito pequena da renda do consumidor, de modo que os consumidores não estariam dispostos a procurar por preços menores a não ser que o diferencial de preço nesta tarefa gerasse uma economia muito elevada. Assim restaria saber o quão grande é este diferencial para o consumidor médio a ponto de justificar esta pesquisa. $O$ valor resultante de uma procura detalhada pelo menor preço resultaria na economia de, no máximo, alguns reais, o que não justificaria o gasto de tempo e dinheiro despendido.

Uma outra hipótese que poderia explicar a assimetria nos preços da gasolina baseia-se na resposta do consumidor as mudanças nos preços da gasolina. Esta resposta pode contribuir para a assimetria entre movimentos nos preços do petróleo e os preços da gasolina no varejo do seguinte modo:

1. Se os consumidores aumentarem suas compras de gasolina para se antecipar a aumentos adicionais quando o preço da gasolina está subindo, então pode ocorrer um aumento da demanda que fará com que o preço suba mais rapidamente;

2. Se os consumidores não retardarem suas compras quando seu preço está caindo tanto quanto aceleraram suas compras quando os preços estavam aumentando então, o preço da gasolina cairá mais lentamente.

Ainda que algumas das teorias expostas acima tentem fornecer uma possível explicação para a assimetria nos preços da gasolina não há consenso sobre a mais adequada. Sendo assim, o que interessa no presente trabalho é averiguar se este fenômeno ocorre no mercado brasileiro.

Para investigar se existe de fato assimetria na resposta dos preços da gasolina a alterações nos preços do petróleo e/ou na taxa de câmbio será necessário o uso de uma ferramenta econométrica que seja aplicável neste caso. No intuito de proceder à análise empírica será utilizado um modelo adequado ao estudo de séries temporais não estacionárias, mais especificamente aquelas que apresentam raiz unitária. Isso porque, como será visto adiante, foi essa a característica encontrada nas variáveis estudadas. Sendo assim, uma forma adequada é utilizar modelos de estimação de variáveis sob co-integração.

\section{MÉTODO DE INVESTIGAÇÃO}

Até finais da década de 70 , os modelos econométricos, baseavam-se no pressuposto de que as séries em estudo eram estacionárias. Uma série não estacionária pode apresentar médias diferentes em diferentes períodos, bem como variância que se aproxima do infinito com o passar do tempo. As conseqüências para as estimações feitas nestas condições são graves pois não é possível obter estatísticas confiáveis, e assim sendo, a análise dos resultados fica desprovida de sentido. Isto é porque a inferência estatística clássica foi desenhada apenas para variáveis que são estacionárias (isto é, com distribuições que não se alteram ao longo do tempo, mantendo ao menos média e variância constante)

Regressões que envolvem processos com tendência estocástica podem dar resultados falsos. Se $y_{t}$ e $x_{t}$ são processos com tendência estocásticade (também conhecidos por prodessos com raiz unitária, que serão definidos adiante) mutuamente independentes, ou seja, $y_{t}$ é independente de $x_{t-j}$ para todo $t$ e $j$, então a regressão via MQO de $y_{t}$ contra $x_{t}$ para $t=1, . ., n$, com ou sem um intercepto, 
resultará numa estimativa em que a probabilidade de se obter um parâmetro significativo (usando-se a distribuição $t$ usual) é maior do que deveria ser. Se $n$ for grande o valor absoluto da estatística $t$ converge em probabilidade para $\infty$ se $n \longrightarrow \infty$. Podería-se concluir então, que $y_{t}$ depende de $x_{t}$, quando na verdade são independentes. Este fenômeno é chamado regressão espúria. Deve-se então tomar cuidado ao analisar séries temporais através de métodos clássicos de inferência. Se a série é um processos de raiz unitária, a aplicação ingênua de tais métodos pode render resultados sem qualquer sentido.

Uma variável é dita ter raíz unitária ou integrada de ordem 1, (denotada por $X_{t} \sim I(1)$ ) se tiver que ser diferenciada para se tornar estacionária. Por conseguinte, se esta variável pode tornar-se estacionária através de uma transformação deste tipo pode-se argumentar que o problema relacionado à sua estimação deveria ser resolvido de forma mais simples, ou seja, bastaria tomar sua diferença para então se obter uma série estacionária. No entanto, tratar séries não-estacionárias através de suas diferenças não é o modo adequado se existe a suspeita de que elas mantêm determinada relação de equilíbrio ao longo do tempo.

Se duas ou mais séries não estacionárias estiverem ligadas por uma combinação de forma que exista uma relação de equilíbrio de longo prazo, então mesmo que isoladamente contenham uma tendência estocástica, elas manterão um percurso bastante próximo ao longo do tempo. Este fato foi denominado de co-integração por Engle e Granger (1987) que mostraram que quando é possível encontrar uma relação estacionária entre séries deste tipo, uma forma adequada de estimação é efetuar uma auto-regressão vetorial em que estejam incluídos os desvios em relação ao equilíbrio de longo prazo.

Se as séries componentes de um determinado sistema forem todas de mesma ordem de integração pode-se efetuar sua estimação através de um modelo de correção erros. Para por em prática este procedimento, toma-se a relação entre variáveis $I(1)$, na forma abaixo:

$$
y_{t}=\alpha+\sum \beta_{i} x_{i t}+\mu_{t}
$$

sendo, $x_{i t}$ as variáveis independentes, $\alpha$ e $\beta_{i}$ parâmetros a serem estimados e $\mu_{t}$ a série dos resíduos a ser estimada, que pode ser serialmente autocorrelacionada e representa os desvios em relação ao equilíbrio de longo prazo entre as variáveis.

Se as séries componentes do sistema descrito acima forem co-integradas então, a regressão de uma delas contra as demais deve produzir uma série de resíduos estacionária. Para verificar a estacionariedade da série de resíduos é possível usar testes de raiz unitária enquanto que as estimativas dos parâmetros podem ser obtidas através de MQO. Assim, deve-se verificar a ordem de integração dos resíduos enfetuando-se sua auto-regressão na forma abaixo:

$$
\Delta \hat{\mu}_{t}=\rho \hat{\mu}_{t-1}+\gamma_{1} \Delta \hat{\mu}_{t-1}+\ldots+\gamma_{p} \Delta \hat{\mu}_{t-p}+\nu_{t}
$$

Logo, se for possível rejeitar a hipótese nula de que $\rho=0$, pode-se concluir pela estacionariedade da série e, portanto pela existência de alguma relação de co-integração. No entanto não é possível utilizar os valores tabelados de Dickey-Fuller para testar essa hipótese. Somente se os verdadeiros valores dos parâmetros da regressão fossem conhecidos de antemão seria possível utilizá-los.

Para contornar este problema MacKinnon (1991) mostrou como desenvolver um experimento de Monte Carlo para gerar os valores críticos adequados ao teste. Eles dependem do tamanho da amostra e do número de variáveis incluídas na regressão. Sendo assim, é possível construir um algoritmo que gere os valores críticos adequados para serem usados nos testes.

Esta metodologia se aplica bem ao caso em que os resíduos da regressão apresentam um comportamento linear. No entanto, diante da possibilidade de existência de um ajustamento ao equilíbrio de longo prazo assimétrico é possível especificar um modelo para os desvios que permita captar melhor o seu comportamento. Caso contrário o teste para averiguar a estacionariedade estará severamente comprometido. 
Além disto, deve-se ter certeza de que o mecanismo de ajustamento da série de resíduos obtidos em (1) seja o mais adequado. Não há, portanto nenhuma garantia de que esta relação tenha de ser linear. Logo, a especificação de um mecanismo não-linear pode ser conveniente para detectar a estacionariedade. Enders e Granger (1998) e Enders e Siklos (2001) mostraram como investigar a possibilidade de que os desvios tenham um comportamento na forma de modelo TAR ou M-TAR.

Para tanto, tomando-se, a série de resíduos na forma de um modelo Threshold Autoregressive (TAR) reescreve-se a Equação (2) na forma abaixo:

$$
\Delta \hat{\mu}_{t}=\rho_{1} I_{t} \hat{\mu}_{t-1}+\rho_{2}\left(1-I_{t}\right) \hat{\mu}_{t-1}+\gamma_{1} \Delta \hat{\mu}_{t-1}+\ldots+\gamma_{p} \Delta \hat{\mu}_{t-p}+\nu_{t}
$$

em que $I_{t}$ é uma variável dummy da forma:

$$
I_{t}=\left\{\begin{array}{lll}
1 & \text { se } & \hat{\mu}_{t-1} \geq \tau \\
0 & \text { se } & \hat{\mu}_{t-1}<\tau
\end{array}\right.
$$

onde $\hat{\tau}$ é o parâmetro chamado threshold que divide o comportamento da série, $\mu_{t}$ a série de resíduos obtidos em (1) e $\nu_{t}$ o resíduo (independente de $\hat{\mu}_{t}$ e de $\tau$ ) assumido ter média zero, variância constante e não apresentar autocorrelação serial. Neste tipo de especificação o vetor de co-integração é linear, mas o modelo de correção de erros é não linear o que abre a possibilidade de que o ajustamento possa se dar de forma descontínua e não-linear Seo (2004).

Alternativamente pode-se tomar uma especificação de forma que a série possa exibir trajetórias mais prolongadas em determinadas direções. Para isto reescreve-se o modelo para o comportamento da séries de resíduos na forma abaixo:

$$
\Delta \hat{\mu}_{t}=\rho_{1} M_{t} \hat{\mu}_{t-1}+\rho_{2}\left(1-M_{t}\right) \hat{\mu}_{t-1}+\gamma_{1} \Delta \hat{\mu}_{t-1}+\ldots+\gamma_{p} \Delta \hat{\mu}_{t-p}+\nu_{t}
$$

em que $M_{t}$ é uma variável dummy da forma:

$$
M_{t}=\left\{\begin{array}{lll}
1 & \text { se } & \Delta \hat{\mu}_{t-1} \geq \tau \\
0 & \text { se } & \Delta \hat{\mu}_{t-1}<\tau
\end{array}\right.
$$

Para que seja possível estimar o modelo de correção de erros conforme as duas especificações acima, após obter os resíduos da estimação da relação de longo prazo deve-se testar a hipótese nula de raiz unitária na série contra a hipótese alternativa de um ajustamento estacionário não-linear. A condição suficiente para que a série seja estacionária foi demonstrada por Petruccelli e Woolford (1984) e consiste em garantir que $-2<\left(\rho_{1}, \rho_{2}\right)<0$.

Faz-se necessário então verificar se $\rho_{1}=\rho_{2}=0$ mas, neste caso (a exemplo do modelo linear), não é possível utilizar os valores tabelados de uma distribuição $F$ padrão no teste. Enders e Granger (1998) mostraram como generalizar a metodologia usada por Dickey e Fuller para testar a hipótese nula de raiz unitária contra a hipótese alternativa de um ajustamento assimétrico.

O procedimento também se baseia num experimento do tipo Monte Carlo, para gerar os valores críticos usados no teste. A princípio deve-se criar, por exemplo, 50.000 processos do tipo random walk da seguinte forma,

$$
y_{k t}=y_{k t-1}+\nu_{k t}
$$

com $k=1, \ldots, K$ se referindo ao número de séries em análise e $t=1, \ldots, T$ o tamanho da amostra. Os valores de $\nu_{k t}$ são obtidos com conjuntos de tamanho $T$ normalmente distribuídos, não-correlacionados e com desvio padrão unitário. Então, tornando-se os valores iniciais de $y_{k t}$ aleatórios, os demais $T$ valores são gerados usando-se a especificação definida em (5).

A próxima etapa é obter uma estimativa dos valores do threshold já que este é desconhecido $a$ priori. Um método para obter uma estimativa superconsistente desses valores $\tau$ foi introduzido por Chan (1993). Para implementá-lo deve-se, a princípio, ordenar a seqüência $\left\{\hat{\mu}_{t-1}\right\}$ em sentido crescente 
considerando apenas os valores contidos no intervalo compreendido entre os $15 \%$ menores e os $15 \%$ maiores como os possíveis candidatos. O passo seguinte é estimar a especificação escolhida (de acordo com (3) ou (4)) com cada valor em potencial de $\tau$, para então selecionar o valor cuja equação geradora retornar a menor soma dos quadrados dos resíduos.

De posse de $\hat{\tau}$, estimam-se as regressões na forma dos modelos (3) ou (4) e para cada uma delas é reportado um valor para a estatística $F$ sob a hipótese nula de que $\rho_{1}=\rho_{2}=0$. Os valores resultantes das estatísticas $F$ obtidos nas 50.000 replicações são tabelados em percentis e denominados $\Phi$ quando o modelo utilizado é o TAR ou $\Phi^{m}$ quando é o $M$-TAR. As Tabelas 4 e 5 apresentam os valores críticos construídos através deste algoritmo.

Obviamente, os valores críticos dependem do tamanho da amostra e do número de variáveis incluídas na estimação. Somando-se a isto o fato de que os modelos assimétricos necessitam da inclusão de um coeficiente adicional, é de se esperar que haja uma perda de poder do teste nestes métodos. No entanto é possível que estes problemas sejam minimizados utilizando-se uma especificação estimada corretamente Enders e Siklos (2001).

O último passo antes da estimação do modelo de correção de erros é inferir sobre a hipótese do ajustamento ser simétrico ou não, ou seja, se $\rho_{1}=\rho_{2}$. No entanto esta tarefa não é das mais fáceis, quando não se conhece o valor de $\tau$. Como é necessário estimá-lo através da auto-regressão dos resíduos, as propriedades de uma distribuição normal multivariada não estão garantidas Chan e Tong (1989).

No entanto, Chan e Tong (1989) mostraram que se $\rho_{1}$ e $\rho_{2}$ forem estimados de forma consistente, ou seja, através método de Chan, pode-se ter condições de normalidade assintoticamente garantidas e, neste caso utilizar o teste $F$ padrão para testar a hipótese de que $\rho_{1}=\rho_{2}$. Com isto testa-se a hipótese de um ajustamento simétrico contra a hipótese alternativa $\rho_{1} \neq \rho_{2}$, ou seja, a possibilidade de que 0 ajustamento seja assimétrico. O número adequado de defasagens a ser utilizado nos testes pode ser obtido através do critério de $\mathrm{AIC}^{1}$ ou $\mathrm{SBC}^{2}$

\section{ANÁLISE EMPÍRICA}

Para desenvolver a análise dos preços da gasolina foram utilizados os seguintes dados:

(i) a taxa de câmbio nominal R\$/U\$,

(ii) o preço de revenda da gasolina e,

(iii) o preço do petróleo no mercado internacional.

Todas as séries estão compreendidas entre julho de 2001 e maio de 2006 perfazendo 59 observações mensais.

A série de preços para a gasolina é disponibilizada pela $\mathrm{ANP}^{3}$ como parte da análise de preços do mercado nacional. Os preços disponibilizados são uma média ponderada da pesquisa feita nos postos de revenda em cada cidade. Os preços do petróleo no mercado internacional foram obtidos no website da Energy Information Administration (EIA) ${ }^{4}$ do governo dos EUA e dizem respeito à média ponderada praticada no mês em questão para o barril de tipo brent cotado em US. A série do dólar refere-se à taxa de câmbio R\$/U\$, comercial para a compra tomando-se sua média mensal ponderada. A fonte é o Banco

\footnotetext{
${ }^{1}$ Akaike Information Criterion, calculado através da fórmula: $[T . \ln .(S Q R)+2 . n]$ sendo, $n$ o número de parâmetros estimados e $T$ o número de observações.

${ }^{2}$ Schwartz Bayesian Criterion, calculado através da fórmula: $[T . \ln .(S Q R)+n \cdot \ln . T]$ sendo, $n$ o número de parâmetros estimados e $T$ o número de observações.

${ }^{3}$ http: //www.anp.gov.br/

${ }^{4}$ http://www.tonto.eia.doe.gov/.
} 
Central do Brasil no Boletim/BP, mas disponibilizada no website do Instituto de Pesquisas Econômicas Aplicadas (IPEA). ${ }^{5}$

Para a estimação as observações foram tomadas com base no mês de maio de 2006 fazendo, $\left(g_{t}\right)$ o logaritmo da série de preços de revenda da gasolina, $\left(o_{t}\right)$ o logaritmo da série de preços do petróleo e, $\left(c_{t}\right)$ o logaritmo da série da taxa de câmbio. A Figura 1 mostra a trajetória das três variáveis no período de tempo em questão.

Figura 1 - Trajetória das séries no tempo

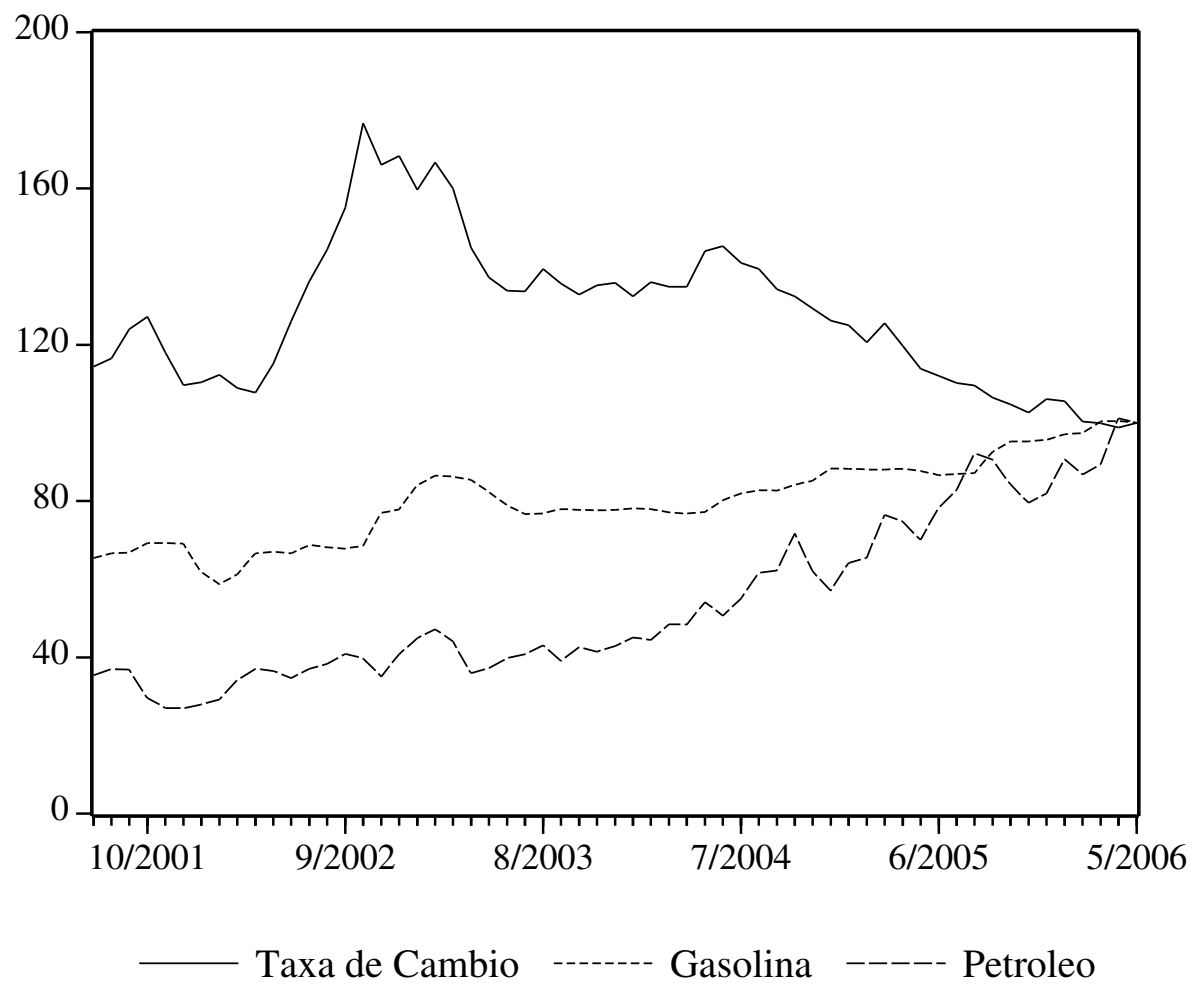

Considerando-se um modelo simples para a formação dos preços da gasolina pode-se utilizar a seguinte especificação:

$$
g_{t}=\alpha+\beta_{1} o_{t}+\beta_{2} c_{t}+\mu_{t}
$$

$\operatorname{com} \alpha, \beta_{1}$ e $\beta_{2}$ parâmetros a serem estimados e $\mu_{t}$ o resíduo a ser estimado. Como é sabido o petróleo tem impacto direto na variação dos preços do combustivel e a taxa de câmbio, como argumentou Bacon (1991) pode contribuir de forma decisiva para a assimetria dos preços dos combustíveis.

\footnotetext{
${ }^{5}$ http://www. ipeadata.gov.br/.
} 
A princípio é preciso testar as séries para verificar sua ordem de integração. Os testes $\mathrm{ADF}^{6}$ mostram que todas as séries possuem raiz unitária e são estacionárias em primeira diferença. Com isto, pode-se dizer, com um nível de confiança de $95 \%$ (usados nos testes), que todas as variáveis se comportam como processos integrados de primeira ordem. Os resultados são apresentados na Tabela 1 e o número de defasagens foi escolhido de acordo com o critério SBC.

Tabela 1 - Testes de raíz unitária

\begin{tabular}{lccc}
\hline Variável & Est. do teste & Valor Crítico & Prob. \\
\hline$g_{t}{ }^{8}$ & $-1,0351$ & $-2,9135$ & 0,7349 \\
$o_{t}{ }^{9}$ & $-2,1093$ & $-3,4970$ & 0,5287 \\
$c_{t}{ }^{8}$ & $-1,2459$ & $-2,9135$ & 0,6485 \\
$\Delta g_{t}{ }^{10}$ & $-5,3274$ & $-1,9467$ & 0,0000 \\
$\Delta o_{t}{ }^{10}$ & $-2,6172$ & $-1,9471$ & 0,0098 \\
$\Delta c_{t}{ }^{10}$ & $-5,4152$ & $-1,9467$ & 0,0000 \\
\hline
\end{tabular}

Pelos resultados acima, é possível perguntar se existe alguma relação de co-integração entre as séries. Para verificar esta possibilidade, num primeiro momento pode-se efetuar o teste de co-integração através do método de Johansen.

\footnotetext{
${ }^{6}$ Augmented Dickey-Fuller. Este teste é uma versão ampliada da forma introduzida por Dickey e Fuller, que permite testar a presença de raiz unitária em séries de ordem mais alta. Pode-se escrevê-lo na forma na forma de (2). Caso o parâmetro $\rho$ seja igual a zero toda a equação e em primeira diferença e, portanto, existe raiz unitária.

${ }^{8}$ A Equação de teste inclui apenas constante.

${ }^{9} \mathrm{~A}$ Equação de teste inclui constante e tendência.

${ }^{10} \mathrm{~A}$ Equação de teste não inclui constante ou tendência.
} 
Como mostra a Tabela 2, a estatística de traço reporta, sob a hipótese de haver ao menos uma relação o valor uma estatística de traço de 7,53 contra 15,49 de valor crítico. Para a hipótese de haver ao menos duas relações de co-integração a estatística apresentada é de 0,02 contra um valor crítico de 3,84. Na estatística de máximo autovalor apura-se que, para a hipótese de que há ao menos uma relação de co-integração o valor da estatística de teste é de 7,50 é o valor crítico de 14,26 . Já para a hipótese de haver duas relações de co-integração o teste apresenta 0,02 contra um valor crítico de 3,84 . Nota-se ainda que, o teste $\lambda_{\max }$ acusa haver uma relação de co-integração com um nível de confiança de $90 \%$.

Tabela 2 - Testes de cointegração: $\lambda_{\text {trace }}$ e $\lambda_{\max }$

\begin{tabular}{lcccc}
\hline$H_{0}$ & $H_{1}$ & $\begin{array}{c}\text { Estatística } \\
\text { de teste }\end{array}$ & $\begin{array}{c}\text { Valor crítico } \\
(5 \%)\end{array}$ & $\begin{array}{c}\text { Valor crítico } \\
(10 \%)\end{array}$ \\
\hline$\lambda_{\text {trace }}$ & \multicolumn{4}{c}{$\lambda_{\text {trace }}$} \\
$r=0$ & $r>0$ & 26,5407 & 29,7971 & 27,0609 \\
$r \leq 1$ & $r>1$ & 7,5306 & 15,4947 & 13,4287 \\
$r \leq 2$ & $r>2$ & 0,0253 & 3,8415 & 2,7055 \\
$\lambda_{\max }$ & & $\lambda_{\max }$ & & \\
$r=0$ & $r=1$ & 19,0101 & 21,1316 & 18,8928 \\
$r=1$ & $r=2$ & 7,5053 & 14,2646 & 12,2965 \\
$r=2$ & $r=3$ & 0,0253 & 3,8415 & 2,7055 \\
\hline
\end{tabular}

Tomando os resultados acima pode-se concluir que não existe relação de co-integração entre as séries. Isto não surpreende uma vez que, como foi argumentado por Enders e Siklos (2001), se as séries forem assimetricamente co-integradas, testes que pressupõem relações lineares entre as variáveis, são mal especificados. A despeito dos resultados obtidos, a intuição econômica diz que deve-se esperar por algum tipo de relação entre as séries. Isso porque o petróleo é o principal componente da gasolina e, portanto deve influenciar o comportamento de seus preços de alguma forma, enquanto que a taxa de câmbio também responde por uma parcela importante das variações dos preços do combustivel.

Adotando agora o método de Engle e Granger para averiguar a existência de relações de co-integração, o próximo passo é regredir as variáveis para obter a relação de equilíbrio de longo prazo. Esta regressão apresenta o seguinte resultado:

$$
g_{t}=2,265+0,343 o_{t}+0,157 c_{t}+\hat{\mu_{t}}
$$

$$
(6,35) \quad(13,96) \quad(2,52)
$$

com estatísticas $t$ mostradas entre parênteses e $\hat{\mu}_{t}$ o resíduo estimado. $O R^{2}$ reportado é de 0,783 enquanto que a estatística de Durbin-Watson é de 0,580. A próxima etapa é verificar a estacionariedade da série de resíduos. Para tanto, deve-se obter a primeira diferença dos resíduos efetuando sua auto-regressão na forma de (2) para determinar a ordem de integração. A forma que respeita as propriedades desejáveis e que se mostrou a mais adequada tanto pelo critério AIC quanto pelo SBC foi a que inclui uma defasagem. Então a seguinte especificação parece ser apropriada:

$$
\begin{gathered}
\Delta \hat{\mu}_{t}=-0,365 \hat{\mu}_{t-1}+0,220 \Delta \hat{\mu}_{t-1}+v_{t} \\
(-3,63) \quad(1,68)
\end{gathered}
$$

De acordo com a auto-regressão acima o valor da estatística $t$ para o coeficiente de interesse é de $-3,63$. A partir destes resultados e com base nos valores críticos mostrados na Tabela 3 não se pode rejeitar a hipótese nula de não-estacionariedade com níveis de confiança de $99 \%$ e $95 \%$. Neste ponto, só seria possível concluir pela co-integração entre as séries se fosse aceito um nível de confiança de $90 \%$. Como este nível não é aceitável para os propósitos deste trabalho não há possibilidade de efetuar a estimação de um modelo de correção de erros. Novamente é provável que isto ocorra em razão da 
pressuposição de um comportamento linear. A possibilidade de que o ajustamento da série de resíduos seja assimétrico torna-se uma opção para a análise.

Tabela 3 - Valores críticos para teste de cointegração linear ${ }^{11}$

\begin{tabular}{lclc}
\hline Percentil & Valor crítico & Percentil & Valor crítico \\
\hline Mínimo & $-6,800855$ & Máximo & 1,446120 \\
$01 \%$ & $-4,531503$ & $99 \%$ & $-0,488035$ \\
$05 \%$ & $-3,873391$ & $95 \%$ & $-1,178953$ \\
$10 \%$ & $-3,551133$ & $90 \%$ & $-1,492615$ \\
$25 \%$ & $-3,028394$ & $75 \%$ & $-1,964571$ \\
\hline
\end{tabular}

Sendo assim como primeira alternativa pode-se optar pelo modelo M-TAR. Então, regredindo a série de desvios do equilíbrio de longo prazo na forma de (4) percebe-se que pelos os valores de $\rho_{1}$ e $\rho_{2}$ mostrados na Tabela $6(-0,407$ e $-0,180$ respectivamente) a série parece ser estacionária. No entanto, o teste para a hipótese de que os coeficientes sejam conjuntamente iguais a zero apresenta uma estatística $F$ de 5,453, o que leva a conclusão, examinando a Tabela 4, de que as séries não são co-integradas. Isso porque, não é possível rejeitar a hipótese nula de raiz unitária nos resíduos em favor de um ajustamento assimétrico já que o valor crítico é de 8,517 com um nível de confiança de $95 \%$.

Tabela $4-\Phi^{m}$ - Valores críticos para teste de cointegração M-TAR ${ }^{12}$

\begin{tabular}{lclc}
\hline Percentil & Valor crítico & Percentil & Valor crítico \\
\hline Mínimo & 0,143056 & Máximo & 23,030930 \\
$01 \%$ & 0,764617 & $99 \%$ & 11,656517 \\
$05 \%$ & 1,339643 & $95 \%$ & 8,516715 \\
$10 \%$ & 1,753043 & $90 \%$ & 7,246599 \\
$25 \%$ & 2,593585 & $75 \%$ & 5,439215 \\
\hline
\end{tabular}

Por fim, resta a especificação na forma de um modelo TAR. Agora, o valor reportado para o teste a de raiz unitária nos resíduos é de 8,58 contra um valor crítico de 8,45 (com 95\% de confiança como mostra a Tabela 5). Neste caso, é possível concluir a favor da estacionariedade da série de resíduos com um mecanismo de ajustamento assimétrico. Sendo assim, pode-se agora reescrever a auto-regressão dos resíduos como:

$$
\begin{aligned}
& \Delta \hat{\mu}_{t}=-0,152 I_{t} \hat{\mu}_{t-1}-1,214\left(1-I_{t}\right) \hat{\mu}_{t-1}+0,203 \Delta \hat{\mu}_{t-1} \hat{v}_{t} \\
& (-2,03) \quad(-3,73) \quad(1,62)
\end{aligned}
$$

em que $I_{t}$ é uma variável dummy da forma:

$$
I_{t}=\left\{\begin{array}{lll}
1 & \text { se } & \hat{\mu}_{t-1} \geq-0,0509 \\
0 & \text { se } & \hat{\mu}_{t-1}<-0,0509
\end{array}\right.
$$

com estatísticas $t$ entre parênteses e $\hat{v}_{t}$ o resíduo estimado. A análise dos valores de $\rho_{1}$ e $\rho_{2}$, indicam estacionariedade na série. Agora a estimativa aponta para convergência da séries de resíduos com uma

\footnotetext{
${ }^{11}$ Os valores críticos incluem constante no vetor de cointegração e foram interpolados de acordo com a metodologia de MacKinnon (1991) para três variáveis e 59 observações.

${ }^{12}$ Os valores críticos incluem constante no vetor de cointegração e foram interpolados de acordo com a metodologia de Enders e Granger (1998) para três variáveis e 59 observações.
} 
velocidade de ajustamento que é mais rápida para discrepâncias positivas do que para as negativas a partir de $\tau=-0,051$.

Tabela $5-\Phi-$ Valores críticos para teste de cointegração $\operatorname{TAR}^{13}$

\begin{tabular}{lclc}
\hline Percentil & Valor crítico & Percentil & Valor crítico \\
\hline Mínimo & 0.183897 & Máximo & 24.244584 \\
$01 \%$ & 0.860878 & $99 \%$ & 11.423222 \\
$05 \%$ & 1.411957 & $95 \%$ & 8.452506 \\
$10 \%$ & 1.797435 & $90 \%$ & 7.181874 \\
$25 \%$ & 2.599235 & $75 \%$ & 5.371948 \\
\hline
\end{tabular}

Tabela 6 - Resumo dos resultados da estimação dos preços da gasolina

\begin{tabular}{lccc}
\hline & Engle e Granger & TAR & M-TAR \\
\hline \multirow{2}{*}{$\rho_{1}{ }^{14}$} & $-0,365$ & $-0,152$ & $-0,478$ \\
& $(-3,63)$ & $(-2,03)$ & $(-3,20)$ \\
$\rho_{2}{ }^{14}$ & - & $-1,214$ & $-0,229$ \\
& & $(-3,72)$ & $(-2,02)$ \\
$\mathrm{AIC}$ & $-124,96$ & $-124,41$ & $-121,51$ \\
$\mathrm{SBC}$ & $-120,87$ & $-120,23$ & $-117,33$ \\
$\rho_{1}=\rho_{2}=0{ }^{15}$ & - & 8,579 & 6,814 \\
$\Phi^{16}$ & & & \\
& $-3,873$ & 8,452 & 8,516 \\
$\rho_{1}=\rho_{2}{ }^{17}$ & & 10,361 & 1,858 \\
& & $(0,002)$ & $(0,178)$ \\
& & &
\end{tabular}

A Tabela 6 mostra as estimações feitas até aqui. Os resultados positivos encontrados com o modelo TAR possiblitam a formulação de um modelo de correção de erros. Assim, a seguinte especificação parece adequada:

$$
\begin{aligned}
& \Delta g_{t}=-0,05 I_{t} \hat{\mu}_{t-1}-0,90\left(1-I_{t}\right) \hat{\mu}_{t-1}+A_{11}(L) \Delta g_{t-1}+A_{12}(L) \Delta o_{t-1}+A_{13}(L) \Delta c_{t-1}+v_{1 t} \\
& (-1,17) \quad(-4,39) \quad\left(F_{11}=0,00\right) \quad\left(F_{12}=0,14\right) \quad\left(F_{13}=0,65\right)
\end{aligned}
$$

\footnotetext{
${ }^{13}$ Os valores críticos incluem constante no vetor de cointegração e foram interpolados de acordo com a metodologia de Enders e Granger (1998) para três variáveis e 59 observações.

${ }^{14}$ Valor estimado com estatísticas $t$ entre parênteses.

${ }^{15}$ Refere-se a hipótese nula $\rho_{1}=\rho_{2}=0$. Valor crítico entre parênteses.

${ }^{16}$ Refere-se tanto ao valor de $\Phi$ quanto de $\Phi^{m}$.

${ }^{17}$ Refere-se a hipótese nula $\rho_{1}=\rho_{2}$. Valor $P$ entre parênteses.
} 


$$
\begin{aligned}
& \Delta o_{t}=-0,10 I_{t} \hat{\mu}_{t-1}+0,05\left(1-I_{t}\right) \hat{\mu}_{t-1}+A_{21}(L) \Delta g_{t-1}+A_{22}(L) \Delta o_{t-1}+A_{23}(L) \Delta c_{t-1}+v_{2 t} \\
& (-1,22) \quad(0,14) \quad\left(F_{21}=0,57\right) \quad\left(F_{22}=0,14\right) \quad\left(F_{23}=0,49\right) \\
& \Delta c_{t}=+0,14 I_{t} \hat{\mu}_{t-1}-0,08\left(1-I_{t}\right) \hat{\mu}_{t-1}+A_{31}(L) \Delta g_{t-1}+A_{32}(L) \Delta o_{t-1}+A_{33}(L) \Delta c_{t-1}+v_{3 t} \\
& (0,82) \quad(-0,11) \quad\left(F_{31}=0,81\right) \quad\left(F_{32}=0,10\right) \quad\left(F_{33}=0,38\right)
\end{aligned}
$$

em que as estatísticas $t$ são apresentadas entre parênteses, $A_{i, j}(L)$ é um polinômio de primeira ordem escrito sob a forma de operadores lag e $F_{i, j}$ é o valor $P$ para a hipótese nula de que todos os valores dos $A_{i, j}$ são nulos.

Outro aspecto que deve ser comentado é que através da inspeção dos resíduos em (9) é possível verificar que não há sinais de autocorrelação serial. No exame dos valores da estatística $Q$ do teste de Ljung-Box ${ }^{7}(Q=6,72$ com um valor $P=0,567)$ percebe-se que estes não são significativos aos níveis exibidos nos resultados.

A Equação (9) mostra que o ajuste na trajetória dos preços da gasolina é de tal forma que, quando os preços caem em relação ao nível de equilíbrio, ou seja $\hat{\mu}_{t-1}<-0,051$, quase $90 \%$ da discrepância é corrigida para o próximo período.

Dado que o modelo escolhido foi da forma TAR, pode-se interpretar os resultados da seguinte maneira: quando ocorre uma realização negativa em $\hat{\mu}_{t}$, ou seja, os preços da gasolina estão abaixo do equilíbrio de longo prazo o retorno ocorre numa velocidade quase dezoito vezes superior a de quando a discrepância é positiva. Alternativamente pode-se dizer que $90 \%$ das realizações negativas são corrigidas de um período para outro contra apenas $5 \%$ das positivas.

Assim a tendência é que aumentos nos preços da gasolina advindos de alterações nos preços do petróleo ou da taxa de câmbio tendem a ocorrer mais rapidamente, enquanto que as quedas tendem a ocorrer mais lentamente. No mais, os valores de $F_{i, j}$ informam que a trajetória de ajuste dos preços da gasolina não é influenciada na sua dinâmica de curto prazo por alterações nos preços do petróleo e na taxa de câmbio. Além disso, dado que o coeficiente de ajustamento estimado para a correção de discrepâncias positivas $\left(\hat{\mu}_{t-1} \geq-0,051\right.$ ) tem uma estatística $t$ de apenas $-1,17$, pode-se dizer que os preços da gasolina não se ajustam quando o ocorre uma realização positiva.

Estes resultados podem ser sintetizados da seguinte forma:

1. Se os preços do petróleo subirem ou taxa de câmbio sofrer uma desvalorização de tal forma que haja uma realização negativa em $\mu_{t-1}$ então é de se esperar que os preços da gasolina subam recuperando $90 \%$ da diferença existente no período subseqüente;

2. Se os preços do petróleo caírem ou taxa de câmbio sofrer uma valorização de tal forma que haja uma realização positiva em $\mu_{t-1}$ (quando seria de se esperar que os preços da gasolina caíssem) então, o ajustamento será de apenas $5 \%$ da diferença existente, ou nem ocorrerá.

Ainda de acordo com a Equação (9) pode-se depreender que tanto os preços do petróleo quanto a taxa de câmbio são fracamente exógenos e não respondem a desvios do equilíbrio de longo prazo como era de se esperar. Além disto, não se pode rejeitar a hipótese de que seus os coeficientes são, em conjunto iguais a zero, o que leva a conclusão que não são influenciados (no sentido da causalidade de Granger) pelas demais variáveis do sistema.

De forma geral esta última especificação mostrou ser a mais adequada tanto pelos resultados alcançados na sua estimação quanto pelos resultados práticos que concordam com a intuição econômica. A estimação por este método também viabilizou uma análise do comportamento dos preços da gasolina, tarefa esta que não foi possível pelo método tradicional e mostrou ter problemas com a especificação na forma de um modelo M-TAR.

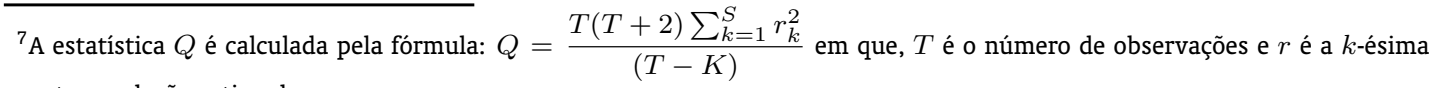
autocorrelação estimada.
} 


\section{CONSIDERAÇÕES FINAIS}

Este trabalho procurou analisar a assimetria no ajustamento dos preços da gasolina em resposta a desvios de sua trajetória de longo prazo para com os preços petróleo no comércio internacional e a taxa de câmbio. Para isto utilizou-se um VAR sob co-integração com um modelo de correção erros de assimétrico e, de acordo com os testes feitos, o modelo de correção de erros mais adequado mostrou ser na forma de um TAR.

Assim, pela metodologia habitual, especificamente os testes de Johansen e o método clássico de Engle e Granger, não foi possível rejeitar a hipótese nula de não haver co-integração entre as séries. Esta hipótese, no entanto, pode ser rejeitada utilizando-se os modelos de co-integração assimétrica. Pelos resultados apresentados comprovou-se a existência de um ajustamento não linear. Desvios da trajetória de equilíbrio mostraram ajustar mais rapidamente para discrepâncias negativas do que para as positivas, ou seja, os preços da gasolina, quando estão acima do equilíbrio, tendem a permanecer em média um período de tempo quase dezoito vezes maior do que quando estão abaixo deste. 


\section{Referências Bibliográficas}

Bachmeier, L. J. \& Griffin, J. M. (2003). New evidence on asymmetric gasoline price responses. Review of Economics and Statistics, 85(3):772-776.

Bacon, R. W. (1991). Rockets and feathers: the asymmetric speed of adjustment of UK retail gasoline prices to cost changes. Energy Economics, 13(3):211-218.

Balke, N. S., Brown, S. P. A., \& Yucel, M. K. (1998). Crude oil and gasoline prices: an asymmetric relationship? Federal Reserve Bank of Dallas Economic Review, 11(First Quarter):2-11.

Bettendorf, L., van der Geest, S. A., \& Varkevisser, M. (2003). Price asymmetry in the Dutch retail gasoline market. Energy Economics, 25:669-689.

Borenstein, S., Cameron, A. C., \& Gilbert, R. (1997). Do gasoline prices respond asymmetrically to crude oil price changes? The Quarterly Journal of Economics, 112(1):305-339.

Chan, K.-S. (1993). Consistency and limiting distribution of the least squares estimator of a threshold autoregressive model. Annals of Statistics, 35(21):520-533.

Chan, K.-S. \& Tong, H. (1989). A Survey of the Statistical Analysis of a Univariate Threshold Autoregressive Model, pages 1-42. JAI Press Inc., Greenwich, Conn.

Enders, W. \& Granger, C. W. J. (1998). Unit-root tests and asymmetric adjustment with an example using the term structure of interest rates. Journal of Business \& Economic Statistics, 16(3):304-311.

Enders, W. \& Siklos, P. L. (2001). Cointegration and threshold adjustment. Journal of Business \& Economic Statistics, 19(2):166-176.

Engle, R. F. \& Granger, C. W. J. (1987). Co-integration and error correction: Representation, estimation and testing. Econometrica, 55(2):251-276.

Galeotti, M., Lanza, A., \& Manera, M. (2003). Rockets and feathers revisited: an international comparison on European gasoline markets. Energy Economics, 25:175-190.

Godby, R., Lintner, A. M., Stengos, T., \& Wandschneider, B. (2000). Testing for asymmetric pricing in the Canadian retail gasoline market. Energy Economics, 22(3):349-368.

MacKinnon, J. (1991). Critical values for cointegration tests. University of California at San Diego, Economics Working Paper Series, 4(90).

Müller, G. \& Ray, S. (2004). Asymmetric price adjustment: A retail perspective.

Peltzman, S. (2000). Prices rise faster than they fall. Journal of Political Economy, 108(3):466-502.

Petrobrás (2005). Destaques operacionais.

Petruccelli, J. D. \& Woolford, S. W. (1984). A threshold ar(1) model. Journal of Applied Probability, 35(21):270-286.

Radchenko, S. (2004). Oil price volatility and the asymmetric response of gasoline prices to oil price increases and decreases. (0408001).

Salas, J. M. I. S. (2002). Price adjustments and asymmetry in the Philippine retail gasoline market. http://ideas.repec.org/p/wpa/wuwpio/0209001.html.

Seo, B. (2004). Testing for nonlinear adjustment in smooth transition vector error correction models. Econometric Society 2004 Far Eastern Meetings, (749). 